PROCEEDINGS OF THE

AMERICAN MATHEMATICAL SOCIETY

Volume 133, Number 1, Pages 191-192

S 0002-9939(04)07531-8

Article electronically published on June 2, 2004

\title{
NONPOSITIVITY: CURVATURE VS. CURVATURE OPERATOR
}

\author{
C. S. ARAVINDA AND F. T. FARRELL \\ (Communicated by Jon G. Wolfson)
}

\begin{abstract}
It is shown that there exist closed Riemannian manifolds $M$ all of whose sectional curvatures are negative, but $M$ does not admit any metric with nonpositive curvature operator.
\end{abstract}

Given a Riemannian manifold $M$, the notion of curvature operator is an important invariant of the Riemannian metric. Recall that the curvature operator at a point $p \in M$ of a manifold $(M,\langle\rangle)$ is a linear map on the space $\Lambda^{2}\left(T_{p} M\right)$ to itself where $T_{p} M$ is the tangent space to $M$ at $p$. If $X, Y, Z, W \in T_{p} M$, then there is a scalar product $\langle$,$\rangle on \Lambda^{2}\left(T_{p} M\right)$ that is given by the formula $\langle X \wedge Y, Z \wedge W\rangle=$ $\langle X, Z\rangle\langle Y, W\rangle-\langle X, W\rangle\langle Y, Z\rangle$ and is extended by linearity to all of $\Lambda^{2}\left(T_{p} M\right)$. Then the curvature operator $\mathcal{R}$ of $M$ is defined by $\langle\mathcal{R}(X \wedge Y), Z \wedge W\rangle:=\langle R(X, Y) W, Z\rangle$ where $R(X, Y) W$ is the Riemann curvature tensor of $M$.

The curvature operator is said to be nonpositive if all its eigenvalues are nonpositive. An elementary linear algebra argument shows that if the curvature operator is nonpositive, then all the sectional curvatures of $M$ are also nonpositive. In his book [3, pp. 239-240], Petersen asks the following.

Question. Are there any compact rank 1 manifolds of nonpositive sectional curvature that do not admit a metric with nonpositive curvature operator?

Recall that any closed Riemannian manifold all of whose sectional curvatures are negative is automatically a rank 1 manifold. Hence the following result shows that the answer to this question is Yes.

Theorem 1. There exist closed Riemannian manifolds whose sectional curvatures are all negative, but which do not admit any Riemannian metric whose curvature operator is nonpositive. In fact, such manifolds exist (at least) in dimensions 8, 16 and 20. Furthermore, given any $\varepsilon>0$, such examples can be constructed (at least in dimension 16) whose sectional curvatures all lie in the interval $[-4,-1+\varepsilon]$.

To exhibit these examples we make use of the following consequence of the superrigidity theorem of K. Corlette [4, Theorem 4.2] (cf. [5, Theorem A] and [6] Theorem 1]).

Theorem 2. Let $M$ be a compact Riemannian manifold that is either quaternionic hyperbolic (of quaternionic dimension at least two) or Cayley hyperbolic. Also, let $N$ be any closed (connected) Riemannian manifold whose curvature operator is

Received by the editors September 18, 2003.

2000 Mathematics Subject Classification. Primary 32Q05, 53C20.

The second author was supported in part by a grant from the National Science Foundation.

(C)2004 American Mathematical Society 
nonpositive. If $M$ and $N$ have isomorphic fundamental groups, then $M$ and $N$ are isometric up to multiplication of the metric by a scalar; in particular, $M$ and $N$ are diffeomorphic.

Given a closed quaternionic hyperbolic manifold of quaternionic dimension 2,4 or 5 (i.e., real dimension 8,16 or 20 ) or a closed Cayley hyperbolic manifold $M$ (whose real dimension is 16), we constructed, in [1] and 2], exotic differential structures carrying negatively curved Riemannian metrics on certain finite covers $\widehat{M}$ of $M$; that is, there exist closed negatively curved manifolds that are homeomorphic but not diffeomorphic to the natural locally symmetric structure on $\widehat{M}$. Applying Theorem 2, we conclude that the exotic differential structures on $\widehat{M}$ cannot support metrics with nonpositive curvature operator. Also, in the case where $M$ is Cayley hyperbolic the sectional curvatures of the Riemannian metric constructed in [1] for the exotic differential structure on $\widehat{M}$ all lie in the interval $[-4,-1+\varepsilon]$. This proves Theorem 1.

\section{REFERENCES}

[1] C.S. Aravinda, F.T. Farrell, Exotic negatively curved structures on Cayley hyperbolic manifolds, Jour. Differential Geom. 63 (2003), 41-62.

[2] C.S. Aravinda, F.T. Farrell, Exotic structures and quaternionic hyperbolic manifolds, to appear in Proc. of the Internat. Conf. on Algebraic groups and Arithmetic, December 17-22, 2001, TIFR, Mumbai.

[3] P. Petersen, Riemannian Geometry, Graduate Texts in Math., 171, Springer-Verlag, NY, 1998. MR98m:53001

[4] K. Corlette, Archimedean superrigidity and hyperbolic geometry, Ann. Math. 135 (1992), 165-182. MR.92m:57048

[5] J. Jost, S.-T. Yau, Harmonic maps and superrigidity, Proc. Sympos. Pure Math., 54, Part 1, 245-280, Amer. Math. Soc., Providence, RI, 1993. MR94m:58060

[6] N. Mok, Y.-T. Siu, S.-K. Yeung, Geometric superrigidity, Invent. Math. 113 (1993), 57-83. MR 94h:53079

Chennai Mathematical Institute, 92, G. N. Chetty Road, Chennai 600 017, India

E-mail address: aravinda@cmi.ac.in

Department of Mathematics, SUny at Binghamton, Binghamton, New York 13902 6000

E-mail address: farrell@math.binghamton.edu 